\title{
Münzen, Lampen, Balsamarien - ihre Rolle im römischen und im provinzialrömischen (treverischen) Bestattungsritus
}

\author{
Elena Köstner
}

\section{Münzen, Lampen und Balsamarien als Indikatoren des Romanisierungspro- zesses in provinzialrömischen Bestattungen?}

Münzen, Lampen und Balsamarien sind aus dem römischen Totenbrauchtum wohl bekannt. Doch auch in provinzialrömischen Bestattungen wurden diese Objekte vor allem nach der römischen Okkupation Galliens gefunden. Inwiefern die indigene Bevölkerung diese Gegenstände in ihrem Totenbrauchtum einsetzte, soll hier am Beispiel der civitas Treverorum verdeutlicht werden. Auch stellt sich die Frage, inwieweit die einheimische Bevölkerung die römisch-italischen Beigabensitten kannte oder ob die so genannten römischen Indikatoren in einen indigen-treverischen Ritus eingebunden wurden. Der Begriff „römische Indikatoren“ umfasst dabei Münzen, Lampen und Balsamarien und wurde deshalb gewählt, weil diese Objekte aus dem römischen Bestattungskontext stammen. In der Forschung werden sie häufig mit einer Romanisierung des Verstorbenen bzw. seiner Angehörigen in Verbindung gebracht. ${ }^{1}$ Es stellt sich die Frage, ob dies auch auf die Bestatteten der civitas Treverorum zutrifft.

Es kann ein allgemeines, übergreifendes Vorgehen nach dem biologischen Tod eines Menschen in verschiedenen antiken Kulturen festgestellt werden: Der Verstorbene wurde gewaschen, neu eingekleidet und aufgebahrt. Klagegesänge wurden angestimmt und der Leichnam wurde umschritten oder umtanzt. Durch diese Handlungen, die von den Angehörigen durchgeführt wurden, sollte der Verstorbene in die Gemeinschaft der Lebenden integriert werden. Häufig schloss sich an die Bestattung ein gemeinsames Mahl an, das zum einen das Zusammengehörigkeitsgefühl betonte und zum anderen der würdigen Verabschiedung des Toten diente. Dieser nahm daran „realiter“ bzw. als sein eigener Gastgeber teil. Der Brauch fand seine Fortsetzung in der Versorgung der Verstorbenen für ihre Reise bzw. ihr Leben im Jenseits mittels Trankund Speisebeigaben. Hintergrund war die Vorstellung des „lebenden Toten“ und eine damit einhergehende Ambivalenz, denn der Verstorbene galt zugleich als vertraut und als unheimlich. Feiertage im Festkalender boten außerdem Gelegenheit, den Toten zu ehren und sich seines Wohlwollens zu versichern. ${ }^{2}$ Dieses Grundmuster bestehend aus Riten zur Vorbereitung des Toten auf die Beisetzung, seiner Versorgung mit Nahrungsmitteln und persönlichem Besitz für das Leben im Jenseits sowie seiner Ehrung an Feiertagen kann als psychologische Reaktion auf den Tod eines Menschen gewertet werden, die sich auch zu einem Automatismus entwickeln konnte. Im Rahmen dieser Handlungen wurden vor allem im Mediterraneum Münzen, Lampen und Balsamarien eingesetzt. Sowohl die rituellen Handlungen als auch die Beigabe von Objekten sind im römischen Totenbrauchtum der republikanischen Zeit und der Kaiserzeit ebenso anzutreffen wie im treverischen Ritus der Spätlatène- und der gallorömischen Zeit.

\footnotetext{
${ }^{1}$ FASOLD 1993, S. 382-387. FASOLD/WITTEYER 1998, S. 181, 189. BÖHME-SCHÖNBERGER 1998, S. 263; REINERT 1998, S. 289f. KAISER 2000, S. 308f. WITTEYER 2000, S. 336.

${ }^{2}$ Exemplarisch seien hier nur die wichtigsten Feste genannt: dies parentales bzw. parentalia, feriae privatae der Caristia oder Cara cognatio, larentalia, lemuria. Detaillierte Informationen liefern beispielsweise SCHRUMPF 2006 und KOLB/FUGMANN 2008.
} 


\section{Römische Indikatoren: Münzen, Lampen, Balsamarien}

Münzen, Lampen und Balsamarien sind Bestandteile des Totengedenkens und keine Beigaben im eigentlichen Sinn. Nach FASOLD und WITTEYER gehören sie zu den „Sekundärbeigaben für die Totenehrung“, wie man sie auch aus dem stadtrömischen Ritus kennt. ${ }^{3}$ Ob sich daraus die These ableiten lässt, dass diese Objekte romanisierte Bestattungen im Sinne eines tief greifenden kulturellen Wandels in provinzialrömischen Bestattungsplätzen kennzeichnen, soll im Verlauf erläutert werden.

In zahlreichen Kulturen wurden den Verstorbenen Münzen beigegeben, wobei nicht alle Geldstücke, die aus Grablegen stammen, als Charonsobolus interpretiert werden können. Sie können auch als Bestandteile des Kleiderbesatzes, als Amulette oder aus Börsen stammend in die Gräber gelangt sein. In den meisten Fällen wurden die Münzen dem zum Zeitpunkt der Bestattung erfolgten Geldumlauf entnommen; trotzdem gelangten auch ältere Exemplare in die Gräber. Die ältesten Befunde dieser Art stammen aus griechischen Bestattungen des 5. Jahrhunderts v. Chr.; dieser Brauch wurde bis in das 5. Jahrhundert $\mathrm{n}$. Chr. in der griechisch-römischen Welt ausgeübt, wobei generell nur wenige Bestattungen Münzbeigaben aufweisen. ${ }^{4}$ Auch in keltischen Gräbern des 3. bis 1. Jahrhunderts v. Chr. stellten sie eine Seltenheit dar. POLENZ postulierte 1982 aufgrund seiner Untersuchung von Münzen in latènezeitlichen Gräbern Mitteleuropas, dass „17 der insgesamt 20 untersuchten Gräber mit Münzbeigaben weibliche Tote“ seien. ${ }^{5}$ Für ihn waren Geldstücke eine Beigabe, die Bestattungen von Frauen kennzeichnen können; dies gilt aber nicht für alle von POLENZ untersuchten Gräber. Diese Annahme trifft aus heutiger Sicht nicht mehr zu. Es existieren verschiedene Interpretationen hinsichtlich der Beigabe von Münzen: „Wurfmünzen“ beschreiben diejenigen Geldstücke, die von den trauernden Angehörigen in das offene Grab geworfen wurden. ${ }^{6}$ Darauf weist die Lage der Münzen im Grab hin. Wenn zwei oder mehr Münzen in der Nähe der Hände oder des Gürtels gefunden wurden, können solche Befunde als „Börsenmünzen“ aufgefasst werden (marsupium). ${ }^{7}$ Sie gehörten also zum persönlichen Besitz des Toten. Der Begriff „parspro-toto-Münzen“ hingegen beschreibt Geldstücke, die stellvertretend für das Vermögen stehen sollten. ${ }^{8}$ Während der Begriff „Börsenmünzen“ lediglich den Inhalt des Geldbeutels bezeichnet, repräsentieren „pars-pro-toto-Münzen“ das Vermögen des Verstorbenen. Sie sind im archäologischen Befund nur aufgrund ihrer Lage im Grab zu unterscheiden, wobei dies bei Brandbestattungen nicht möglich ist. Die Interpretation einer einzelnen Münze im Grab als Obolus für Charon ist wohl die bekannteste. ${ }^{9}$ Der Fährmann soll die Toten über die Unterweltflüsse an das Tor des Hades bringen, wofür ihm ein Lohn zusteht. Verschiedene Schriftquellen berichten, dass die Münze in den Mund des Verstorbenen gelegt wurde. ${ }^{10}$ Charon forderte nur einen geringen Betrag für seine Dienste; THÜRY spricht von ein bis zwei Münzen. ${ }^{11}$ STEVENS nennt weitere Kennzeichen für Charonsmünzen: „(1) a single low-denomination coin (2) is placed in the mouth (3) at the time of death (4) to pay Charon’s fee. “12 Jedoch treffen

\footnotetext{
${ }^{3}$ FASOLD/WITTEYER 1998, S. 189.

${ }^{4}$ STEVENS 1991, S. $223 f$.

${ }^{5}$ POLENZ 1982, S. 165.

${ }^{6}$ THÜRY 1999, S. 20; GORECKI 1975, S. 276-278.

${ }^{7}$ GORECKI 1975, S. 250.

${ }^{8}$ THÜRY 1999, S. 20.

${ }^{9}$ THÜRY 1999, S. 17f. Aristoph. Ran. 139-142; 270f. Anth. Pal. 11,209; 11,171. Lukian Charon 11.

${ }^{10}$ GORECKI 1975, S. 236f. Apul. met. 6,18f. Iuv. 2,149-152; 3,264-267. Lukian De luctu 3; 10.

11 THÜRY 1999, S. 27-30.

${ }^{12}$ STEVENS 1991, S. 216.
} 
nicht alle Aspekte auf provinzialrömische Befunde zu: In der civitas Treverorum wurden zwischen 150 v. und 100/120 n. Chr. vornehmlich Kremationen durchgeführt. Die Lage der Münzen im bzw. am toten Körper kann nicht mehr eruiert werden und somit ist STEVENS zweites Kennzeichen nicht nachprüfbar. ${ }^{13}$ Münzen, die in Brandbestattungen gefunden wurden, weisen nur dann Verbrennungsspuren auf, wenn sie gemeinsam mit dem Leichnam kremiert wurden. Es besteht auch die Möglichkeit, dass die Geldstücke nach der Verbrennung in die Urne bzw. auf den Leichenbrand in der Grabgrube gelegt wurden. Auch STEVEns vierter Aspekt bleibt für die Treverer zu bezweifeln, denn der Glaube an Charon war zwar bis in die römische Kaiserzeit weit verbreitet und lebendig, doch gibt es keine Hinweise darauf, inwieweit dieser in der civitas Treverorum praktiziert wurde. „The evidence shows that ,Charon's Obol' was only one manifestation of a much wider funerary use of coins and suggests a richer and broader context in which it can be understood." ${ }^{14}$ GORECKI nimmt an, dass auch die Kelten den Brauch der Münzbeigabe kannten, obwohl dieser nur in geringem Umfang ausgeübt wurde; doch erst durch die römische Vermittlung habe diese Sitte in den Nordwestprovinzen eine gewisse Bedeutung erhalten. ${ }^{15}$

Neben der Beigabe von Münzen ist aus dem römischen Kontext außerdem die Verwendung von Öllampen im Rahmen von Totengedenkfeiern bekannt. Die Öllampe hat ihren Ursprung im östlichen Mittelmeerraum und fand zwischen dem 9. und 6. Jahrhundert v. Chr. durch die Phöniker im gesamten Mediterraneum Verbreitung. ${ }^{16}$ Zunächst waren es einfache Tonschälchen mit einem mehrmals eingedrückten Rand, um den Docht besser fassen zu können. Daraus entwickelte sich ab dem 7. Jahrhundert v. Chr. der geschlossene Lampenkörper, der nur noch ein Loch für den Docht frei ließ. Zu diesem Zeitpunkt setzte in Athen auch die Produktion der Lampen auf der Drehscheibe ein. ${ }^{17}$ Auf der italischen Halbinsel wurde etwa ab augusteischer Zeit ein neuer Lampentypus hergestellt, der sich von den hellenistischen Formen unterschied, die mit Hilfe von Matrizen gefertigt worden waren. Die neuen, italischen Lampen wiesen eine Volutenschnauze und eine flachere Oberseite auf; dadurch schuf man ausreichend Platz für bildliche Darstellungen. ${ }^{18}$ Außerdem wies die eingesenkte Deckelplatte ein kleines Loch zum Einfüllen des Öls auf. Die Lampen verfügten an ihrer Spitze über ein Dochtloch. Der Schnauze gegenüber befand sich der Lampenhenkel. Im Gegensatz zum Mittelmeerraum wurden in den nördlichen Provinzen des imperium Romanum aber auch Talglampen verwendet, wogegen die römischen Öllampen meist mit Olivenöl betrieben wurden. Sowohl Olivenöl als auch Öllampen gelangten wohl nur selten in die Zone nordwärts der Alpen, denn im archäologischen Befund der Späthallstatt- und Frühlatènezeit fehlen sie. Die älteste bekannte Lampe aus der civitas Treverorum wurde in dem Kammergrab von Clemency entdeckt; dieses Exemplar wurde aus Italien importiert. ${ }^{19}$

Außerdem wurden im mediterranen Totenbrauchtum auch Balsamarien bzw. Unguentarien verwendet. In solchen Gefäßen wurden meist medizinische oder kosmetische Produkte wie Salben und Öle aufbewahrt. Aus Gräbern und Siedlungen sind verschiedene Gefäßformen bekannt. ANDERSON-STOJANOVIC unterscheidet zwei

\footnotetext{
13 THÜRY 1999, STEVENS 1991 und GORECKI 1975 und 1995 beschäftigten sich in erster Linie mit Körperbestattungen, da sich diese besser für ihre Untersuchungen eigneten. Ihre Ergebnisse lassen sich jedoch nur bedingt auf Kremationen übertragen.

${ }^{14}$ STEVENS 1991, S. 215.

${ }^{15}$ GORECKI 1995, S. 102.

${ }^{16}$ RADT 1986, S. 41.

${ }^{17}$ RADT 1986, S. 45f.

${ }^{18}$ RADT 1986, S. 53.

${ }^{19}$ METZLER/WARRINGO/BIS/METZLER-ZENS 1991, S. 95
} 
Haupttypen: „1) The fusiform or spindle shape with body varying from rounded to slender, and a foot usually set off clearly from the body, 2) the bulbous shape with round or pear-shaped body and flat base.“" ${ }^{\prime 20}$ Sowohl kleine Miniaturen von vier bis fünf Zentimetern Höhe als auch solche Exemplare von 20 bis 30 Zentimetern Höhe wurden in Gräbern entdeckt. Der schmale Gefäßkörper und die weite Öffnung boten sich an für ein langsames und sparsames Ausgießen von Flüssigkeiten. Außerdem sollte wohl durch die kleine Öffnung verhindert werden, dass Luft in großen Mengen in das Innere des Behälters gelangte und die darin enthaltenen Duftstoffe nicht so schnell entweichen konnten. Aufgrund der mangelhaften Dichtung der Gefäße konnten sich die Aromen der Salben und Öle jedoch schnell verflüchtigen.

\section{Charakteristika des römischen Totenbrauchtums anhand ausgewählter Beispiele}

Was man über das römische Totenbrauchtum weiß, bezieht sich meist auf Italien und betrifft in erster Linie die Totenfeierlichkeiten der stadtrömischen Oberschicht. Die Abläufe sind nicht ohne Weiteres auf andere Regionen bzw. Bevölkerungsteile übertragbar. Die römischen Bestattungsbräuche haben Parallelen zu etruskischen Zeremonien, d. h. aber nicht, dass man sie völlig aus dem etruskischen Ritual ableiten kann. Für manche Sitten existierte eine panitalische Tradition.

Die Nekropolen von Ostia bieten die Möglichkeit, die Bestattungstraditionen vom 2. Jahrhundert v. Chr. bis in die frühe Kaiserzeit zu betrachten; der Fokus soll hier jedoch auf die Verwendung der so genannten römischen Indikatoren gelegt werden: An der via Ostiensis befinden sich die ältesten Grablegen. Die Brandbestattungen wurden zwischen der 2. Hälfte des 2. und der 1. Hälfte des 1. Jahrhunderts v. Chr. angelegt. $^{21}$ Der Leichenbrand wurde meist in einfachen Urnen deponiert. Neben keramischem Trinkgeschirr, Schmuck und Totenklinen, die auf einen hellenistischen Einfluss verweisen, wurden auch Münzen, Lampen und Balsamarien niedergelegt; nicht alle dort bestatteten Personen erhielten all diese Gegenstände. ${ }^{22}$ In frühaugusteischer Zeit wurde die Anzahl der beigegebenen Objekte in der Nekropole von Ostia reduziert. ${ }^{23}$ Dieses Phänomen konnte auch in anderen Bestattungsplätzen der italischen Halbinsel beobachtet werden und ist auf den Monopolanspruch des Augustus und der kaiserlichen Familie zurückzuführen. Mit der zunehmenden Gleichmachung der Gesellschaft in der frühen Kaiserzeit wurden die politischen Konkurrenzkämpfe der Eliten unterdrückt und es kam zu einer Standardisierung der Gräber. Die repräsentative Selbstdarstellung durch die sepulkrale Architektur blieb der kaiserlichen Familie vorbehalten. Die columbaria der spätaugusteisch-tiberischen Zeit aus Ostia tragen dieser Entwicklung Rechnung. Die moderatere äußere Gestaltung der Grablegen wurde durch eine aufwendige Ausstattung des Inneren der Anlage kompensiert, beispielsweise mit grabeigenen ustrina, Herden und Brunnen für Totenbankette etc. ${ }^{24}$ Es zeichnete sich eine Abkehr von der Öffentlichkeit und der nach außen getragenen Selbstdarstellung ab sowie die Hinwendung zur familia im Inneren der Grabarchitektur. Die Beigabenausstattung erscheint nun standardisiert: Beispielsweise wurden ein kleiner Napf, ein bis zwei Miniaturen und Miesmuschelschalen niedergelegt; das et-

\footnotetext{
${ }^{20}$ ANDERSON-STOJANOVIC 1987, S. 106.

${ }^{21}$ HEINZELMANN 1998, S. 42.

${ }^{22}$ HEINZELMANN 1998, S. 43f.

${ }^{23}$ HEINZELMANN 1998, S. 45.

${ }^{24}$ HEINZELMANN 1998, S, 46.
} 
was reichere Inventar bestand zum Beispiel aus zwei Glasbalsamarien, Glaspaste, Lampen, einem Napf und vier größeren Muschelschalen. ${ }^{25}$

Die Entwicklung der Beigabensitte in Ostia, wie sie Heinzelmann beschreibt, ist nicht ohne Weiteres auf andere Regionen Italiens übertragbar; in Norditalien beispielsweise zeichnete sich ein anderes Bild ab. Es konnten unterschiedliche Entwicklungsprozesse beobachtet werden, die darauf zurückgeführt werden können, dass hier verschiedene ethnische Gruppen lebten: Die Nekropolen der Transpadana und des Veneto, die seit der späten Republik durchgehend belegt waren, verweisen auf eine einheimische Bevölkerung (S. Maria di Zevio, S. Materno a Dorno, Gropello Cairoli und Nave). ${ }^{26} \mathrm{Ab}$ augusteischer Zeit konnte hier jedoch ein Wandel in der Ausstattung der Gräber beobachtet werden. Während das Inventar der spätrepublikanischen Brandbestattungen vor allem aus einheimischem Tongeschirr, Münzen, Fibeln und Gerätschaften bestand, traten ab augusteischer Zeit erstmals auch Lampen und Balsamarien auf, die man vorher in Zusammenhang mit diesen Bestattungsplätzen nicht kannte. ${ }^{27}$ Außerdem konnte beobachtet werden, dass die Beigabe einheimischer Keramik zu diesem Zeitpunkt rückläufig war. Indigene Fibelformen fehlten dann um die Mitte des 1. Jahrhunderts n. Chr. ${ }^{28}$ Das stadtrömische Totenbrauchtum - hier wurde es am Beispiel Ostias verdeutlicht - breitete sich ab der frühen Kaiserzeit nach Mittelund Norditalien aus. FASOLD und WITTEYER bringen dies mit der Ansiedlung von Veteranen aus dem römischen Bürgerkrieg nach der Schlacht von Actium (31 v. Chr.) in Zusammenhang; diese gelten ihrer Meinung nach als Vermittler der römischen Totenehrung und der Nutzung von Lampen und Balsamarien. ${ }^{29}$

\section{Römische Indikatoren und ihre Verwendung in den Gräbern der civitas Treverorum}

Wie erwähnt gelten Münzen, Lampen und Balsamarien aus Glas oder Ton in der Forschungsliteratur oftmals als Anzeichen für eine Romanisierung des Totenbrauchtums. Die römischen Indikatoren besitzen einen heterogenen Charakter: Münzen sind zwar zu den sekundären Beigaben zu zählen, wurden aber beispielsweise auch im Rahmen des Ahnenkults als Objekte des Totengedenkens am geschlossenen Grab deponiert (vgl. Grab 14 aus Goeblingen-Nospelt). ${ }^{30}$ Balsamarien und Lampen konnten ebenso in der Grube deponiert werden oder auch außerhalb des geschlossenen Grabs. ${ }^{31}$

Aus der civitas Treverorum (in den Grenzen von $85 \mathrm{n}$. Chr.) wurden insgesamt 950 Bestattungen der Nekropolen Lamadelaine und Feulen (Großherzogtum Luxemburg), Lebach „Die Motte“ (Kr. Saarlouis, Saarland), Hoppstädten-Weiersbach (Kr.

\footnotetext{
${ }^{25}$ HEINZELMANN 1998, S. 46.

${ }^{26}$ FASOLD/WITTEYER 1998, S. 188.

${ }^{27}$ FASOLD/WITTEYER 1998, S. 184-189.

${ }^{28}$ FASOLD/WITTEYER 1998, S. $185 \mathrm{f}$.

${ }^{29}$ FASOLD/WITTEYER 1998, S. 189.

${ }^{30}$ METZLER/GAENG/LeGOFF 2009, S. 451-453. In der Hügelaufschüttung von Grab 14 wurden kleine Gruben mit Überresten von Speisebeigaben und verbrannten Münzen entdeckt. Diese Gruben wurden nach der Bestattung angelegt. Die in ihnen gefundenen Tierknochen und Münzen waren verbrannt worden (S. 138). Sie wurden im Rahmen der Riten des Totengedenkens angelegt. Weitere Münzen stammen aus einem dolium in der Hügelaufschüttung: Diese 58 Geldstücke stammen aus dem Zeitraum zwischen dem vorletzten Jahrzehnt v. Chr. und der Mitte des 2. Jahrhunderts n. Chr. Das zeigt, dass noch lange nach dem Tod der Frau Erinnerungsriten an ihrem Grab durchgeführt wurden (S. 138140).

${ }^{31}$ FASOLD 1993, S. $382 f$.
} 
Bernkastel-Wittlich, Rheinland-Pfalz) und Wederath-Belginum (Kr. Birkenfeld, Rheinland-Pfalz) hinsichtlich ausgewählter Objekte der Grabinventare untersucht. ${ }^{32}$ Dazu gehören u. a. auch die so genannten römischen Indikatoren. Ergänzt wird die Materialbasis durch herausragende Bestattungen: Clemency, Livingen, Hellingen, Goeblingen-Nospelt Grab A, B, C, D, 14, 20 (Großherzogtum Luxemburg), Olewig Grab 3 (Stadt Trier, Rheinland-Pfalz) und Elchweiler-Schmißberg Grab A und B (Kr. Birkenfeld, Rheinland-Pfalz). Um die Untersuchungsergebnisse besser strukturieren zu können, wurde der Untersuchungszeitraum (150 v. bis 100/120 n. Chr.) in drei Phasen unterteilt. Diese Arbeitshilfe dient lediglich der Synchronisierung der einzelnen Belegungsstufen der Nekropolen und stellt keine Alternative zu bestehenden Chronologien dar. Phase 1 umfasst den Zeitraum von 150 bis 30/20 v. Chr. Dazu zählen die Stufen Lt D1a bis Lt D2b, die aus Lamadelaine, Feulen und Wederath-Belginum bekannt sind, sowie die Belegungsphasen 1-4 aus Hoppstädten-Weiersbach und der Horizont „caesarische Zeit“ aus Lebach. Hinzu kommen die herausragenden Bestattungen von Clemency (Lt D2a), Elchweiler-Schmißberg Grab A (Lt D2a), Olewig Grab 3 (Lt D2a/b) und Goeblingen-Nospelt Grab C, D und 20 (Lt D2b). Phase 2 beinhaltet den Zeitraum zwischen 30/20 v. und 10/20 n. Chr. Dazu gehören die Horizonte GR1 und GR2 (Lamadelaine und Feulen), die Zeitstufe 2 aus Lebach, die Belegungsphase 5 aus Hoppstädten-Weiersbach sowie die Frauenphasen 1-4 und die Männerphase 1 aus Wederath-Belginum. Hierzu zählen auch die Grablegen von Geoblingen-Nospelt Grab A, B und 14 (GR1) sowie Livingen (GR2) und ElchweilerSchmißberg Grab B (GR2). Phase 3 (10/20 bis 100/120 n. Chr.) umfasst schließlich die römische Periode, die in Lamadelaine nicht mehr vorkommt; in Feulen wird diese mit R1 und R2 beschrieben. Dazu zählen außerdem die Zeitstufen 3-5 aus Lebach, die Belegungsphasen 6 und 7 aus Hoppstädten-Weiersbach sowie die Frauenphasen 5-8 und die Männerphasen 2 und 3 aus Wederath-Belginum. Hinzu kommen die beiden Bestattungen aus Hellingen (Grab A datiert in R1 und Grab B in R2).

In Phase 1 (150 bis 30/20 v. Chr.) war die Münzbeigabe in Lamadelaine, Feulen und Wederath-Belginum in geringem Umfang ausgeübt worden (in 3\%, 5\% bzw. $1 \%$ der Gräber wurden Geldstücke gefunden). ${ }^{33}$ Womöglich kann hier auf einen vorrömischen Ritus der Münzbeigabe geschlossen werden. In Lebach und Hoppstädten-Weiersbach wurden zu dieser Zeit keine Geldstücke in den Gräbern deponiert; dies gilt auch für die herausragenden Bestattungen von Clemency, Olewig Grab 3 und Elchweiler-Schmißberg Grab A sowie Goeblingen-Nospelt Grab C, D und 20. In Phase 2 (30/20 v. bis 10/20 n. Chr.) stieg die Anzahl der Gräber mit Geldstücken in Lamadelaine und Wederath-Belginum auf 13\% bzw. 27\%. ${ }^{34}$ Dies kann womöglich darauf zurückgeführt werden, dass in der Nähe der betreffenden Siedlungen auch römische Militärlager existierten. Eventuell kam es hier zu einer Beeinflussung der indigenen Bestattungsbräuche, als deren Resultat mehr Münzen beigegeben wurden. ${ }^{35}$ Ob hier von einer römischen Beeinflussung der Bestattungsbräuche gesprochen werden kann, bleibt aber fraglich. Es war zwar zu einer römischen Einflussnahme gekommen, doch muss von einer eher oberflächlichen Beeinflussung ausgegangen

\footnotetext{
${ }^{32}$ Vgl. KÖSTNER 2011, S. 91-192. Alle Werte, die hier angeführt werden, entstammen dieser Untersuchung. Es wurden nur vollständige Bestattungen (d. h. mit menschlichen Überresten) aufgenommen. Außerdem wurden nur vollständig rekonstruierbare Objekte gewertet; einzelne Scherben von Gefäßen, die nicht sicher zugeordnet werden konnten, blieben unberücksichtigt.

${ }^{33}$ KÖSTNER 2011, S. 102f., 114f, 157-163, $215 f$.

${ }^{34}$ KÖSTNER 2011, S. 102f., 157-163, 215.

${ }_{35}$ Auf dem Titelberg existierte vom Ende des gallischen Krieges bis in augusteische Zeit ein römisches Militärlager (GASPAR 2007, S. 20); auch in Wederath-Belginum bestand ein römisches Lager von 30 v. bis 50/70 n. Chr. (OLDENSTEIN 2000, S. 28).
} 
werden, denn sonst überwogen das spätlatènezeitliche Inventar und die spätkeltischen Bestattungssitten. Während in Lebach die Münzbeigabe in geringem Ausmaß einsetzte (7\% der Gräber), wurde in Hoppstädten-Weiersbach nur in dieser Phase eine Münze beigegeben, was 9\% der Gräber entspricht. ${ }^{36}$ In Feulen wurden in Phase 2 keine Münzen in den Gräbern platziert; dies gilt auch für die herausragenden Bestattungen von Livingen, Elchweiler-Schmißberg Grab B, Goeblingen-Nospelt Grab A, B und 14. Für Phase 3 (10/20 bis 100/120 n. Chr.) kann ein erneuter Anstieg der Zahl der Gräber mit Münzbeigabe verzeichnet werden und zwar in Feulen auf 7\% und in Wederath-Belginum auf 52\% der Gräber. ${ }^{37}$ Während in Lebach die Anzahl der Bestattungen mit Münzen gleich blieb (7\% der Gräber), wurden in Hoppstädten-Weiersbach keine Münzen beigegeben. ${ }^{38}$ Auch in den herausragenden Bestattungen von Hellingen (Grab A und B) wurden zu keiner Zeit Geldstücke in den Gräbern deponiert.

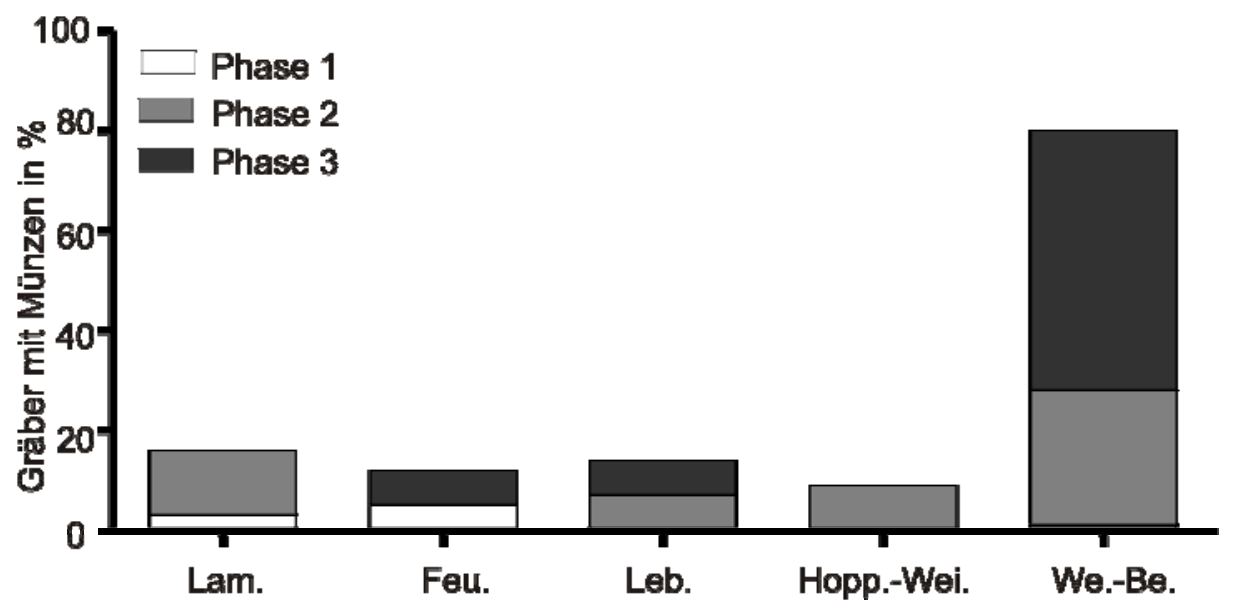

Abb. 1 Anteil der Gräber mit Münzen aus den untersuchten Gräberfeldern (in \%).

Generell fehlen Münzen in den herausragenden Bestattungen, obwohl die Mehrheit von ihnen (vgl. Clemency, Livingen, Elchweiler-Schmißberg Grab B, GoeblingenNospelt Grab A, B, C, D, 14 und 20, Hellingen Grab A und B) in unterschiedlichem Ausmaß Importe aus dem Mittelmeerraum aufwies. Anscheinend waren diese Verstorbenen bzw. ihre Angehörigen aufgeschlossener gegenüber neuen Produkten. Außerdem besaßen sie die nötigen finanziellen Mittel und die Kontakte zu Händlern, um an derartige Objekte zu gelangen. ${ }^{39}$ Sie nutzten diese Importe, um ihren gesellschaftlichen Status darzustellen. Im Gegensatz dazu weisen die Bestattungen aus den Nekropolen nur selten importierte Keramikerzeugnisse auf: In Phase 1 wurden nur in Wede-

\footnotetext{
${ }^{36}$ KÖSTNER 2011, S. 125f., 137, $215 f$.

${ }^{37}$ KÖSTNER 2011, S. 114, 157-163, 215.

${ }^{38}$ KÖSTNER 2011, S. 137, 215.

${ }^{39}$ Während die Siedlungen von Feulen und Hoppstädten-Weiersbach nicht in unmittelbarer Nähe zu römischen Fernstraßen lagen, wiesen diejenigen von Lamadelaine, Lebach und Wederath-Belginum eine deutlich verkehrsgünstigere Lage auf. Sowohl der Titelberg (Lamadelaine und Clemency) als auch der vicus von Dalheim (Livingen, Hellingen, Goeblingen-Nospelt) befanden sich in der Nähe der Fernstraße Arlon-Trier. Die Siedlung von Lebach profitierte von der Lage an der Route Metz-Bingen und Wederath-Belginum von den Fernstraßen Trier-Mainz sowie der Verbindung zwischen Eifel, Moselund Nahetal. Während sich die Bestattungen von Olewig im Einzugsgebiet des civitas-Hauptortes Augusta Treverorum befanden, führte bei den Gräbern von Elchweiler-Schmißberg die Route TrierMainz vorbei.
} 
rath-Belginum importierte Keramikwaren genutzt und zwar in $0,4 \%$ der Gräber. $^{40}$ In Phase 2 trifft dies auf 3\% der Gräber aus Lamadelaine und 1,5\% der Gräber aus Wederath-Belginum zu. ${ }^{41}$ In Phase 3 enthielten 0,8\% der Gräber aus Feulen, 8\% der Lebacher Gräber, 3,5\% der Bestattungen aus Hoppstädten-Weiersbach und 3\% der Gräber aus Wederath-Belginum Importkeramik. ${ }^{42}$

Außerdem konnte festgestellt werden, dass in den fünf untersuchten Gräberfeldern nicht mehr als vier Münzen in die Gräber gelangten. ${ }^{43}$ Es ist also nicht anzunehmen, dass Geldstücke der Repräsentation von Reichtum dienten. Es überwogen diejenigen Bestattungen, die nur eine einzelne Münze enthielten. Doch ist es äußerst unwahrscheinlich, dass diese als Lohn für den Fährmann Charon niedergelegt wurden, da es keine weiteren schriftlichen Belege dafür gibt, dass diese Glaubensvorstellung in der civitas Treverorum von der einheimischen Bevölkerung praktiziert wurde. Durch die Betrachtung der verschiedenen Münztypen, die in den Gräbern gefunden wurden, können Hinweise darauf gefunden werden, in welchem zeitlichen Rahmen die Niederlegung von Geldstücken erfolgte. Dabei muss berücksichtigt werden, dass, obwohl die Mehrheit der beigegebenen Münzen dem momentanen Geldumlauf entnommen wurden, auch alte Geldstücke in die Gräber gelangt sein konnten.

\begin{tabular}{l|l} 
Münztyp & Anzahl \\
\hline keltische Prägungen & $\mathbf{1 2}$ \\
\hline Augustus & $\mathbf{7 3}$ \\
\hline Agrippa & $\mathbf{3}$ \\
\hline Tiberius & $\mathbf{2 9}$ \\
\hline Caligula & $\mathbf{2 7}$ \\
\hline Claudius & $\mathbf{2 0}$ \\
\hline Nero & $\mathbf{4}$ \\
\hline Vespasian & $\mathbf{1 0}$ \\
\hline Titus & $\mathbf{2 8}$ \\
\hline Domitian & $\mathbf{5}$ \\
\hline Nerva & $\mathbf{1}$ \\
\hline Traian & $\mathbf{1}$ \\
\hline nicht identifizierbare Münzen & $\mathbf{3 7}$
\end{tabular}

Tab. 1 Anzahl der in den Gräbern deponierten Münztypen aus allen Belegungsphasen bzw. Gräberfeldern.

Tab. 1 zeigt, dass es sich bei den meisten Prägungen um solche aus der Zeit des Augustus handelt. Es kam also in diesem zeitlichen Rahmen zu einem deutlichen Anstieg der Münzbeigabe. Dabei ist zu berücksichtigen, dass 70 der 73 augusteischen Münzen aus Wederath-Belginum stammen. ${ }^{44} \mathrm{Ab}$ ca. $30 \mathrm{v}$. Chr. existierte ein römisches Militärlager in Wederath-Belginum. Eventuell kam es hier zu einer römischen Beeinflussung der spätkeltischen Bestattungssitten. Dieser Impetus erstreckte sich jedoch nur auf die Kategorie Münzen, denn wie noch gezeigt werden wird, gelangten Lampen und Balsamarien nur in geringer Anzahl in die Gräber aus Wederath-Belginum. Für die folgenden Jahrzehnte konnte eine kontinuierliche Fortsetzung der Münzbeigabe in geringem Umfang in der civitas Treverorum beobachtet werden. Während für die Regierungszeit des Nero ein deutlicher Rückgang verzeichnet werden kann, er-

\footnotetext{
${ }^{40}$ KÖSTNER 2011, S. 145.

${ }^{41}$ KÖSTNER 2011, S. 96, 145.

${ }^{42}$ KÖSTNER 2011, S. 107, 118f., 130, 145, 206-208.

${ }^{43}$ KÖSTNER 2011, S. 215.

${ }^{44}$ KÖSTNER 2011, S. 214.
} 
holte sich der Wert dann für Münzen aus der Regierungszeit des Vespasian und des Titus. ${ }^{45}$ Gegen Ende des 1. Jahrhunderts n. Chr. wurde die Beigabe von Geldstücken nur noch in sehr geringem Umfang ausgeübt.

Lampen gelangten weitaus seltener in die Gräber der untersuchten Nekropolen als Münzen. ${ }^{46}$ In Lamadelaine, Feulen, Lebach und Hoppstädten-Weiersbach fehlen sie gänzlich in den Phasen 1 und 2. In Wederath-Belginum waren sie in den Phasen 2 und 3 in sehr geringem Ausmaß vorhanden (in 1,5\% bzw. 2\% der Gräber). ${ }^{47}$ In Feulen und Lebach gelangten Lampen erst in Phase 3 in die Gräber und zwar in 7\% bzw. 2\% der Bestattungen. ${ }^{48}$ Während die Öllampe aus Goeblingen-Nospelt Grab B in Phase 2 datiert wird, gehört das Exemplar aus Clemency in Phase $1 .^{49}$

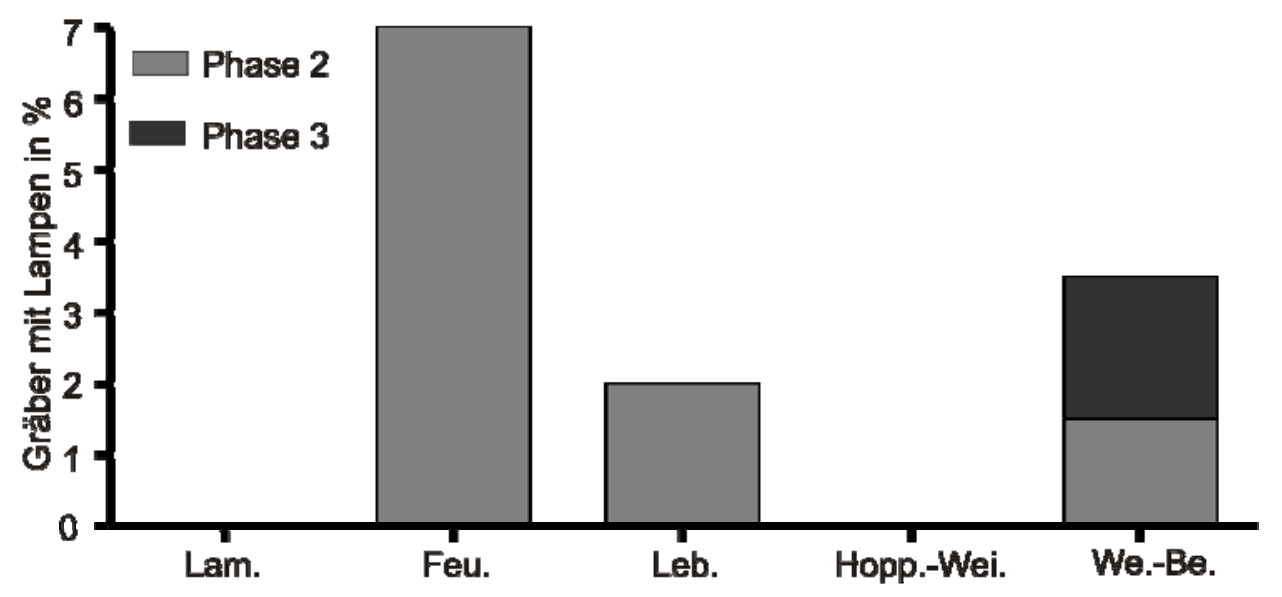

Abb. 2 Anteil der Gräber mit Lampen aus den untersuchten Gräberfeldern (in \%).

Balsamarien wurden in Phase 1 nicht in den Gräbern deponiert. In HoppstädtenWeiersbach fehlen sie vollständig während der gesamten Nutzung des Bestattungsplatzes. In Lamadelaine gelangten Salbgefäße in Phase 2 in 9\% der Gräber, in Feulen in $7 \%$, in Lebach in $10 \%$ und in Wederath-Belginum in $0,5 \%$ der Gräber. ${ }^{50}$ Außerdem wurde ein Exemplar in Grab B aus Elchweiler-Schmißberg gefunden. In Phase 3 traten sie in Feulen in 18,5\% der Gräber auf, in Lebach in 8\% und in Wederath-Belginum in $4 \%$ der Gräber sowie in den beiden Hellinger Bestattungen. ${ }^{51}$

\footnotetext{
${ }^{45}$ KÖSTNER 2011, S. 214.

${ }^{46}$ Die in den untersuchten Gräbern gefundenen Lampen stammen nicht immer aus der civitas Treverorum, sondern in vielen Fällen wurden sie importiert wie beispielsweise die Öllampe aus Clemency, die wahrscheinlich aus Campanien stammt. (METZLER/WARRINGO/BIS/METZLER-ZENS 1991, S. 92f.).

${ }^{47}$ KÖSTNER 2011, S. 161, $215 f$.

${ }^{48}$ KÖSTNER 2011, S. 114f., 126, $215 f$.

${ }^{49}$ METZLER/WARRINGO/BIS/METZLER-ZENS 1991, S. 50. METZLER/GAENG/LeGOFF 2009, S. 105. KÖSTNER 2011, S. 171, 182.

${ }^{50}$ KÖSTNER 2011, S. 102, 114f., 126, 162f., 215f. Während tönerne Balsamarien meist aus einheimischer Produktion stammen - wie z. B. die beiden Exemplare aus Lamadelaine (METZLER/METZLER-ZENS/MENIEL/BIS/GAENG/VILLEMAR 1999, S. 337) -, trifft dies auf Salbgefäße aus Glas nicht immer zu.

${ }^{51}$ KÖSTNER 2011, S. 114f., 126, 162f., 173f, 215f.
} 


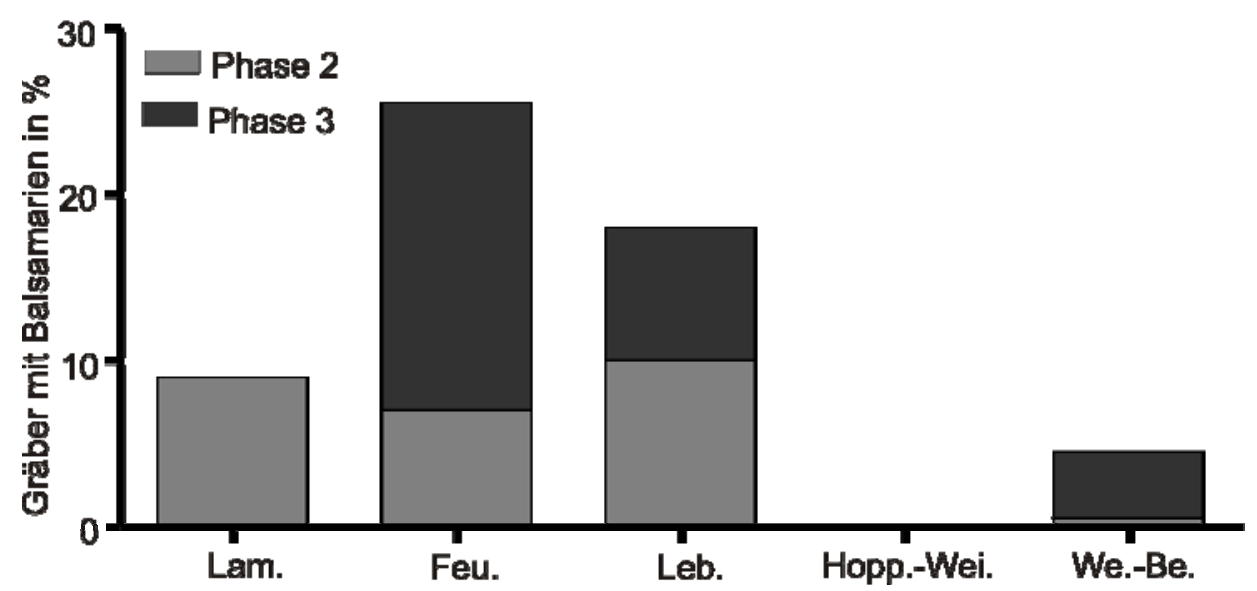

Abb. 3 Anteil der Gräber mit Balsamarien aus den untersuchten Gräberfeldern (in \%).

\section{Fazit: Bewusste Übernahme römischer Riten und deren Attributen oder lediglich eine sinnentleerte Adaption?}

Lampen und Balsamarien entstammen den römischen Beigabensitten und haben keine Vorläufer im keltischen (treverischen) Totenbrauchtum. Diese Objekte gelangten frühestens um 30 v. Chr. in die Gräber von Lamadelaine, Feulen und Lebach bzw. zwischen $20 \mathrm{v}$. und $20 \mathrm{n}$. Chr. in die Bestattungen von Hoppstädten-Weiersbach und Wederath-Belginum. Im Gegensatz dazu wurden Münzen bereits in Grablegen der spätkeltischen Zeit in den Nekropolen von Lamadelaine, Feulen und Wederath-Belginum (ab 150 v. Chr.) gefunden. Deshalb kann auf einen ähnlichen Brauch in der Spätlatènekultur geschlossen werden. Jedoch wurden Geldstücke auch in Heiligtümern niedergelegt. Eventuell kannte die einheimische Bevölkerung wenigstens grundlegende Aspekte des römisch-italischen Beigabenwesens. Eine sinnentleerte Adaption ist dabei nicht ausgeschlossen, vor allem dann nicht, wenn man berücksichtigt, dass sonst das Inventar spätkeltischen Prinzipien folgte wie z. B. die Beigabe von zahlreichen keramischen Gefäßen, Waffen und Gerätschaften, Fibeln und Schmuck. Die These einer sinnentleerten Adaption wird außerdem dadurch unterstrichen, dass nur in wenigen Fällen eine Vergesellschaftung von Münzen, Lampen und Balsamarien, wie sie auch aus dem römischen Totenbrauchtum bekannt ist, vorliegt. Während eine derartige Kombination in Lamadelaine, Hoppstädten-Weiersbach und in den herausragenden Bestattungen nicht festgestellt werden konnte, wurden in Feulen, Lebach und Wederath-Belginum derartige Zusammenstellungen beobachtet. Grab 87 aus Feulen enthält eine tönerne Öllampe und vier keramische Balsamarien. ${ }^{52}$ Die Gräber 62 und 11 aus Lebach weisen jeweils eine Münze und ein gläsernes Balsamarium auf. ${ }^{53}$ Aus Wederath-Belginum stammen sechs Bestattungen mit dieser Kombination: Grab 1026 (drei Münzen und zwei gläserne Balsamarien), Grab 1227 (zwei Münzen sowie ein gläsernes Balsamarium), Grab 2212 (eine Münze und ein gläsernes Balsamarium), Grab 2215 und 1898 (jeweils zwei Münzen und ein gläsernes Balsamarium). ${ }^{54}$ Allerdings wurden in keinem Fall alle drei Elemente der römischen Indikatoren in einem Grab deponiert.

\footnotetext{
${ }^{52}$ SCHENDZIELORZ 2006, S. 78.

53 GERLACH 1986, S. 18f., 52f.

${ }^{54}$ HAFFNER 1978, S. 26f., 72f. CORDIE-HACKENBERG/HAFFNER 1997, S. 15, 82f., 122-124.
} 
Aufgrund der selektiven Deponierung römischer Indikatoren in treverischen Gräbern muss man nicht zwangsläufig veränderte Glaubensvorstellungen annehmen. Auch kann im Zeitraum bis 100/120 n. Chr. durch ihre Verwendung nicht zweifelsfrei auf romanisierte Bestattungen im Sinne eines tief greifenden kulturellen Wandels geschlossen werden. Lediglich auf die Hellinger Gräber A und B sowie auf ElchweilerSchmißberg Grab B und Goeblingen-Nospelt (Grab A, B und 14) trifft zu, dass durch einen römischen Impetus ausgelöste Veränderungen in diesen Grablegen stärker aufgegriffen wurden. Ein Beispiel dafür, dass sich einige Bewohner der civitas Treverorum eher als Römer denn als Kelten sahen, ist die Darstellung einer Kampfszene auf einem Reliefblock aus Bartringen (Großherzogtum Luxemburg): Diese zeigt vier Menschen und ein Pferd während eines Kampfes; außerdem sind zwei zu Boden gefallene Schilde und Lanzen sowie „der am Boden liegende Barbar [...] in seltsam verdrehter Körperhaltung“ abgebildet. ${ }^{55}$ Die Figur rechts des Einheimischen hält diesen an den Haaren; an der Armbeuge dieser Figur, die als römischer Soldat zu interpretieren ist, hängt ein Torques, der als Kriegsbeute gedeutet wird. ${ }^{56}$ Das Grabmonument wird auf 20/30 n. Chr. datiert und KREMER sieht in Zusammenhang mit der villa von Bartringen den Sitz einer romtreuen, romanisierten Familie. ${ }^{57}$ Die Schlachtszene drückt ihrer Meinung nach eine „antikeltische Haltung“ aus. ${ }^{58}$ Doch muss das Nebeneinander von keltischer Identität und prorömischer Haltung nicht immer mit Konflikten behaftet gewesen sein. Während der Reliefblock mit Kampfszene aus Bartringen und einige der herausragenden Bestattungen einen stärkeren römischen Impetus erkennen lassen, kann für die Mehrheit der Bevölkerung, die in den Bestattungsplätzen von Lamadelaine, Feulen, Lebach, Hoppstädten-Weiersbach und Wederath-Belginum ihre letzte Ruhe fand, angenommen werden, dass bei der Verwendung der so genannten römischen Indikatoren einem Modetrend nachgegangen wurde und daher eher eine sinnentleerte Adaption vorliegt als die genaue Kenntnis römischer Beigabensitten und Jenseitsvorstellungen.

\footnotetext{
${ }^{55}$ KREMER 2009, S. 79.

${ }^{56}$ KREMER 2009, S. 81.

${ }^{57}$ KREMER 2009, S. 101, 105-107, 112.

${ }^{58}$ KREMER 2009, S. 107.
} 


\section{Literaturverzeichnis}

ANDERSON-STOJANOVIC, V. R. 1987, The Chronology and Function of Ceramic Unguentaria, in: AJA 91, S. 105-122.

BÖHME-SCHÖNBERGER, A. 1998, Das Gräberfeld von Badenheim, in: P. Fasold (Hrsg.), Bestattungssitte und kulturelle Identität. Grabanlagen und Grabbeigaben der frühen römischen Kaiserzeit in Italien und den Nordwestprovinzen, Xantener Berichte, Grabung - Forschung - Repräsentation 7, Köln, S. 261-283.

CORDIE-HACKENBERG, R./HAFFNER, A. 1991-1997, Das keltisch-römische Gräberfeld von Wederath-Belginum, Bd. 4-5, Mainz.

FASOLD, P. 1993, Romanisierung und Grabbrauch: Überlegungen zum frührömischen Totenkult in Rätien, in: M. Struck (Hrsg.), Römerzeitliche Gräber als Quellen zu Religion, Bevölkerungsstruktur und sozialgeschichtlicher Archäologie, Schriften des Instituts für Vor- und Frühgeschichte der Johannes Gutenberg-Universität Mainz Bd. 3, Mainz, S. 381-394.

FASOLD, P./WITTEYER, M. 1998, „Römisches“ in den Gräbern Mittel- und Norditaliens, in: P. Fasold (Hrsg.), Bestattungssitte und kulturelle Identität. Grabanlagen und Grabbeigaben der frühen römischen Kaiserzeit in Italien und den Nordwestprovinzen, Xantener Berichte, Grabung - Forschung - Repräsentation 7, Köln, S. 293304.

GASPAR, N. 2007, Die keltischen und gallorömischen Fibeln vom Titelberg, Dossiers d'Archéologie du Musée National d'Histoire et d'Art XI, Luxemburg.

GERLACH, G. 1986, Das Gräberfeld „Die Motte“ bei Lebach, 2 Bde., Saarbrücker Beiträge zur Altertumskunde Bd. 15, Bonn.

GLESER, R. 2005, Studien zu sozialen Strukturen der historischen Kelten in Mitteleuropa aufgrund der Gräberanalyse, Die keltisch-römische Nekropole HoppstädtenWeiersbach im Kontext latènezeitlicher Fundgruppen und römischer Okkupation, Saarbrücker Beiträge zur Altertumskunde Bd. 81, Bonn.

GORECKI, J. 1975, Münzbeigabe in römerzeitlichen Körpergräbern, in: BRGK 56, S. 179-467.

GORECKI, J. 1995, Die Münzbeigabe, eine mediterrane Grabsitte. Nur Fährlohn für Charon? in: Witteyer, M./Fasold, P. (Hrsg.), Des Lichtes beraubt, Totenehrung in der römischen Gräberstraße von Mainz-Weisenau, Wiesbaden, S. 93-103.

HAFFNER, A. 1978, Das keltisch-römische Gräberfeld von Wederath-Belginum, Bd. 3, Mainz.

HEINZELMANN, M. 1998, Die Nekropolen von Ostia: Zur Entwicklung der Beigabensitte vom 2. Jh. v. Chr. bis in die frühe Kaiserzeit, in: P. Fasold (Hrsg.), Bestattungssitte und kulturelle Identität. Grabanlagen und Grabbeigaben der frühen 
römischen Kaiserzeit in Italien und den Nordwestprovinzen, Xantener Berichte, Grabung - Forschung - Repräsentation 7, Köln, S. 179-192.

KAISER, M. 2000, Elemente der Romanisierung im Grabbrauch des 1. Jahrhunderts n. Chr. in der Augusta Treverorum, in: A. Haffner/S. v. Schnurbein (Hrsg.), Kelten, Germanen, Römer im Mittelgebirgsraum zwischen Luxemburg und Thüringen, Kolloquien zur Vor- und Frühgeschichte Bd. 5, Bonn, S. 305-317.

KÖSTNER, E. 2011, Tod im Trevererland, Interkulturelle Beziehungen zwischen Römern und Kelten, Eine historisch-archäologische Gräberanalyse in der civitas Treverorum zwischen 150 v. und 100/120 n. Chr., Pietas Bd. 3, Gutenberg.

KOLB, A./FUGMANN, J. 2008, Tod in Rom, Grabinschriften als Spiegel römischen Lebens, Mainz.

KREMER, G. 2009, Das frühkaiserzeitliche Mausoleum von Bartringen (Luxemburg), Dossiers d'Archéologie du Musée National d'Histoire et d'Art XII, Luxemburg.

METZLER, J./WARRINGO R./BIS, R./METZLER-ZENS, N. 1991, Clemency et les tombes de l'aristocratie en Gaule Belgique, Dossiers d'Archéologie du Musée National d'Histoire et d'Art I, Luxemburg.

METZLER, J./METZLER-ZENS, N./MENIEL, P./BIS, R./GAENG, C./VILLEMAR, I. 1999, Lamadelaine, Une nécropole de l'oppidum du Titelberg, Dossiers d’Archéologie du Musée National d’Histoire et d’Art VI, Luxemburg.

METZLER, J./GAENG, C./LeGOFF, I. 2009, Goeblange-Nospelt, Une nécropole aristocratique trévire, Dossiers d'Archéologie du Musée National d'Histoire et d'Art XIII, Luxemburg.

OLDENSTEIN, J. 2000, Wederath-Belginum, Gräberfeld, Lager, Siedlung und Tempelbezirk, in: A. Haffner/S. von Schnurbein (Hrsg.), Kelten, Germanen und Römer im Mittelgebirgsraum zwischen Luxemburg und Thüringen, Kolloquien zur Vor- und Frühgeschichte, Bd. 5, Bonn, S. 23-29.

POLENZ, H. 1982, Münzen in latènezeitlichen Gräbern Mitteleuropas, in: BVBI 47, S. 27-222.

RADT, W. 1986, Lampen und Beleuchtung in der Antike, in: Antike Welt 17, S. 4158.

REINERT, F. 1998, „Römisches“ in treverischen Gräbern der julisch-claudischen Zeit, in: P. Fasold (Hrsg.), Bestattungssitte und kulturelle Identität. Grabanlagen und Grabbeigaben der frühen römischen Kaiserzeit in Italien und den Nordwestprovinzen, Xantener Berichte Grabung - Forschung - Repräsentation 7, Köln, S. 285-294.

SCHENDZIELORZ, S. 2006, Feulen, Ein spätlatènezeitlich-frührömisches Gräberfeld in Luxemburg, Dossiers d'Archéologie du Musée National d'Histoire et d’Art IX, Luxemburg. 
SCHRUMPF, S. 2006, Bestattung und Bestattungswesen im römischen Reich, Ablauf, soziale Dimension und ökonomische Bedeutung der Totenfürsorge im lateinischen Westen, Bonn.

STEVENS, S. T. 1991, Charon's Obol and Other Coins in Ancient Funerary Practice, in: Phoenix 45, S. 215-229.

THÜRY, G. E. 1999, Charon und die Funktion der Münzen in römischen Gräbern der Kaiserzeit, in: O. Dubuis/S. Frey-Kupper/G. Perret (Hrsg.), Trouvailles monétaires de tombes - Fundmünzen aus Gräbern, Lausanne, S. 17-30.

WITTEYER, M. 2000, Grabgestaltung und Beigabenausstattung in der Gräberstraße von Mainz-Weisenau, in: A. Haffner/S. v. Schnurbein (Hrsg.), Kelten, Germanen, Römer im Mittelgebirgsraum zwischen Luxemburg und Thüringen, Kolloquien zur Vor- und Frühgeschichte Bd. 5, Bonn, S. 319-343.

\section{Kontakt zur Autorin:}

Elena Köstner

elena.koestner@gmx.de 